\title{
Impact of plasma turbulence on linear mode conversion of quasi-optical wave beams in toroidal magnetic traps
}

\author{
$\underline{T}^{\text {T. A. Khusainov }}{ }^{1}$, A. G. Shalashov ${ }^{1}$, E. D. Gospodchikov ${ }^{1}$, and A. Köhn² \\ ${ }^{1}$ Institute of Applied Physics of Russian Academy of Sciences, Nizhny Novgorod, Russia, hta@appl.sci-nnov.ru \\ ${ }^{2}$ Universität Stuttgart, IGVP, Stuttgart, Germany
}

\section{Introduction}

Linear transformation of the ordinary $(\mathrm{O})$ and extraordinary $(\mathrm{X})$ electromagnetic modes in the vicinity of the plasma cutoffs in magnetized plasma is a basis for advanced schemes of microwave heating and diagnostic of over-dense plasmas (where the central density exceeds the cutoff) in magnetic fusion research [1]. On the other hand, plasma density fluctuations are typical of magnetic fusion experiment.

In this work, we investigate the influence of density fluctuations on the process of linear O-X mode coupling in non-one-dimensional magnetized plasma, using analytical approaches and full-wave numerical simulations.

We limit our studies to a two-dimensional model of the coupling region, having in mind that taking into account the actual three-dimensional structure of the magnetic field and the curvature of the magnetic surfaces in tokamaks and stellarators does not lead to a qualitative change in the equations describing the linear transformation [3].

\section{Analytical model}

We assume that the primary influence of density fluctuations on the $\mathrm{O}-\mathrm{X}$ mode coupling efficiency is due to a small-angle scattering along the whole trace of a microwave beam, while the influence of the fluctuations on the tunneling process itself (inside the coupling region) can be neglected [2]. Such scattering leads to an additional phase modulation of the wave beam, but not to the refraction or reflection of the beam as a whole. Thus, we arrive at the following statement of the problem - a random wave beam that has experienced the influence of fluctuations along the path falls on the regular domain of the $\mathrm{O}-\mathrm{X}$ mode coupling.

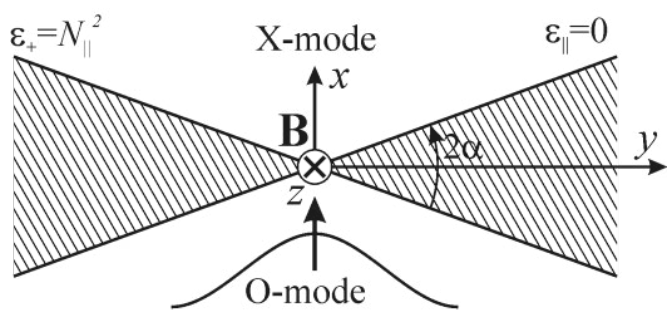

Fig. 1. Coordinate system and the evanescent region (shown by hatching) for the left-hand polarized plane electromagnetic waves with given the refractive index along the magnetic field $N_{\|}=c k_{\|} / \omega$.

The structure of the coupling region for the twodimensional model is shown in Fig. 1. O-wave tunnels through the evanescent area formed by or intersecting the cutoff planes $\varepsilon_{+}(x, y)=N_{\|}^{2}$ and $\varepsilon_{\|}(x, y)=0$. Here $\varepsilon_{+}$and $\varepsilon_{\|}$are the components of the dielectric permittivity tensor in the Stix representation,

$$
\varepsilon_{\|}=1-n_{\mathrm{e}} / n_{\mathrm{cr}}, \quad \varepsilon_{+}=1-\left(n_{\mathrm{e}} / n_{\mathrm{cr}}\right) /\left(1+B / B_{\text {res }}\right),
$$

where $n_{\text {cr }}=m_{\mathrm{e}} \omega^{2} /\left(4 \pi e^{2}\right)$ is the critical plasma density for the wave frequency $\omega$ and $B_{\text {res }}=m_{\mathrm{e}} c \omega / e$ is the magnetic field corresponding to the electron cyclotron resonance.

The coefficient of the O-X mode conversion can be defined as the fraction of the electromagnetic energy flux tunneling through the evanescent region. It was shown, that for the chosen geometry, provided that length of the beam trace is less than the characteristic diffraction length, the coupling efficiency $\langle T\rangle$, averaged over the ensemble of fluctuations, can be calculated as

$$
\langle T\rangle=\int_{-\infty}^{\infty} G\left(y, y^{\prime}\right) \tilde{A}(y) \widetilde{A}^{*}\left(y^{\prime}\right) \Phi\left(y-y^{\prime}\right) d y d y^{\prime},
$$

Here $G$ is the known Green function [3] defining two-dimensional structure of the mode coupling region, $\widetilde{A}(y)$ is the deterministic distribution of the complex wave amplitude and function $\Phi(\Delta y)$ takes into account the additional random phase modulation $\phi(y)$ and is defined as follows:

$$
\Phi\left(y, y^{\prime}\right)=\exp \left(-\left\langle\phi^{2}\right\rangle+\left\langle\phi(y) \phi\left(y^{\prime}\right)\right\rangle\right) .
$$

For the small-angle scattering, the random phase is related to the density fluctuations $\delta n_{\mathrm{e}}$ as

$$
\phi(x, y)=\int_{x_{0}}^{x} \beta\left(x^{\prime}\right) \delta n_{\mathrm{e}}\left(x^{\prime}\right) d x^{\prime}, \quad \beta=\frac{\partial k_{\perp}}{\partial n_{\mathrm{e}}},
$$

where $\beta$ determines the dependence of the component of the wave vector transverse to the magnetic field on the plasma density.

As a result, the phase correlation function is related to the density correlation function as follows:

$$
\begin{aligned}
& \left\langle\phi(x, y) \phi\left(x, y^{\prime}\right)\right\rangle=\int_{x_{0}}^{x} \int_{x_{0}}^{x} d x^{\prime} d x^{\prime \prime} \times \\
& \quad \times \beta\left(x^{\prime}\right) \beta\left(x^{\prime \prime}\right)\left\langle\delta n_{\mathrm{e}}\left(x^{\prime}, y\right) \delta n_{\mathrm{e}}\left(x^{\prime \prime}, y^{\prime}\right)\right\rangle .
\end{aligned}
$$

Using this equation we meet some uncertainty related to the particular choice of the upper limits of integration. In a simplest theory we may just assume $x=0$, however, in the neat vicinity of the transformation region, $x \rightarrow 0$, the assumption about smallangle scattering may become inapplicable as diminishing values of $k_{\perp}$ result in a saturation of the spectral broadening for $x>x_{\mathrm{m}}$ [2]. To estimate this effect, one may assume that the diffusive broadening stops when the spectral width $\left\langle\delta k^{2}\right\rangle$ becomes compa- 
rable with the solution $k_{\perp}^{2}$ of the dispersion relation for the $\mathrm{O}$ wave propagating in the unperturbed plasma. Thus, the upper limits $x=x_{\mathrm{m}}$ in (3) are defined as a negative root of the following equation

$$
\left\langle\delta k^{2}\left(x_{\mathrm{m}}\right)\right\rangle=k_{\perp}^{2}\left(x_{\mathrm{m}}\right), \quad x_{\mathrm{m}}<0 .
$$

\section{Comparison with the full-wave calculations}

To verify our analytical theory, we carried out a direct numerical simulation of the tunneling of the incident $\mathrm{O}$ wave through the two-dimensionally inhomogeneous layer of a fluctuating overdense plasma. Maxwell equations were solved with the full-wave finite-difference time-domain code IPF-FDMC [4].

According to our basic model, the magnetic field and plasma density is assumed to vary in $(x, y)$ plane. Their regular profiles inside the simulation domain $\left[-x_{0}, x_{0}\right] \times\left[-y_{0}, y_{0}\right]$ is selected to reproduce the two-dimensional model shown in Fig. 1:

$$
\begin{aligned}
& n_{\mathrm{e}} / n_{\mathrm{cr}}=\left(1+\tanh \frac{x \cos \alpha-y \sin \alpha}{L_{n}}\right) \tanh \frac{x+x_{0}}{L_{n} / 3}, \\
& B / B_{\text {res }}=Y-2(Y+1) y \sin \alpha / L_{n} .
\end{aligned}
$$

The distribution of density fluctuations is calculated as a sum over a discrete set of harmonics,

$$
\begin{aligned}
& \delta n_{\mathrm{e}} / n_{\mathrm{cr}}=A \exp \left(-\left(x-x_{\delta n}\right)^{2} /\left(2 l_{\delta n}^{2}\right)\right) \times \\
& \sum_{\kappa_{x}, \kappa_{y}} \exp \left(-\frac{\kappa_{x}^{2}+\kappa_{y}^{2}}{8} \lambda^{2}\right) \cos \left(\kappa_{x} x+\kappa_{y} y+\xi\left(\kappa_{x}, \kappa_{y}\right)\right) .
\end{aligned}
$$

Here $A$ is the normalization factor; $l_{\delta n}$ and $x_{\delta n}$ characterize size and position of the fluctuating area. For each realization, a sum over $200 \times 100$ harmonics with random phases $\xi\left(\kappa_{x}, \kappa_{y}\right)$ is generated, then the results of electromagnetic simulations are averaged over 100 realizations.

To demonstrate the effect of fluctuations, we fix the width of the incident beam and study the dependence of the averaged $\mathrm{O}-\mathrm{X}$ coupling efficiency on the width $l_{\delta n}$ of the region with density perturbations, see Fig 2. Here we compare the results of numerical simulations of $\langle T\rangle$ with the analytical calculations based on equations (1)-(4) for both the onedimensional (a) and two-dimensional (b) geometries.

Solid lines correspond to the theory with saturation of the small-angle scattering being taken into account and dotted lines correspond the simplest theory without saturation, i.e. in (3) we take $x=x_{\mathrm{m}}$ in the first case, and $x=0$ in the second case. Both theories coincide to each other and to the numerical modeling when the density fluctuations are localized far enough from the mode-coupling region $l_{\delta n}<<\left|x_{\delta n}\right|$. When fluctuations are present inside the coupling region $l_{\delta n} \sim\left|x_{\delta n}\right|$, the saturation results in $\sim 10 \%$ variation of $\langle T\rangle$. Note also, for the chosen beam size in twodimensional geometry, the $\mathrm{O}-\mathrm{X}$ coupling efficiency is higher than those for the same beam in onedimensional case.

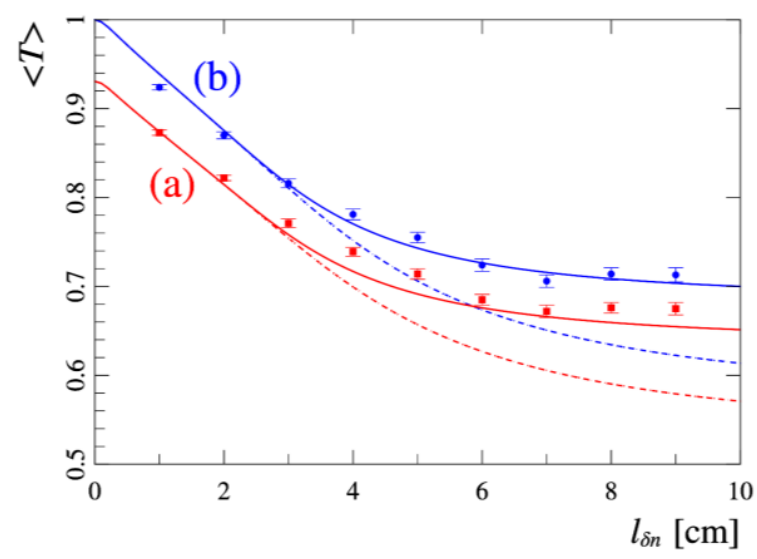

Fig. 2. The $\mathrm{O}-\mathrm{X}$ mode coupling efficiency $\langle T\rangle$ of the Gaussian beam with the fixed width $a_{y}=3 \mathrm{~cm}$ depending on the size $l_{\delta n}$ of the region occupied by fluctuations for (a) $\alpha=0$ and (b) $\alpha=0.05$. Lines show predictions of the analytical theory with (solid lines) and without (dotted lines) the saturation of small-angle scattering being taken into account. Points correspond to full-wave numerical simulations; the errorbars of the numerical results are due to statistical dispersion after averaging over 100 random realizations. Other parameters of the model are: $k_{0}=12 \mathrm{~cm}^{-1}$, $\delta n_{\mathrm{e}} / n_{\mathrm{cr}}=3 \%, \quad x_{\delta n}=-6 \mathrm{~cm}, \lambda=0.5 \mathrm{~cm}, \quad L_{\nabla}=0.7 \mathrm{~cm}$, $Y=0.8, L_{n}=10 \mathrm{~cm}, x_{0}=10 \mathrm{~cm}, y_{0}=16 \mathrm{~cm}$.

\section{Summary}

We considered the effect of a random phase modulation induced by density fluctuations along the beam propagation path on the $\mathrm{O}-\mathrm{X}$ mode coupling efficiency in a two-dimensional geometry. In this case, the averaged coupling efficiency could be expressed in a universal way as a convolution of deterministic functions characterizing the geometry of the scattering region, the regular part of the beam shape, and the correlation function of the distribution of the random phase, see (1). The analytical results are verified with a full-wave code. We find a relatively good agreement of both approaches, and demonstrate the role of two physical effects related to non-onedimensionality of the non-perturbed plasma and stopping of the stochastic spectral broadening of the wave beam near the $\mathrm{O}-\mathrm{X}$ mode coupling region. Both effects modify the coupling efficiency by about $10 \%$ in a parameter range typical of magnetic fusion experiment and should be taken into account.

Acknowledgements. The work was done under Russian state assignment (projects Nos. 0035-20140027, 0035-2018-0003) and was supported by RFBR (pr. 18-42-520069).

\section{References}

1. Laqua, H. P. // Plasma Phys. Control. Fusion 2007. V. 49. No 4. P. R1.

2. Shalashov, A. G. and Gospodchikov, E. D. // Plasma Phys. Control. Fusion 2014. V. 56. P. 125011.

3. Khusainov, T. A., Shalashov, A. G. and Gospodchikov, E. D. // Plasma Phys. Rep. 2018. V. 44, No 5. P. 484.

4 Köhn, A. et al // Plasma Phys. Control. Fusion 2008. V. 50. P. 085018 